\title{
Implementación de caudalímetro tipo flotámetro en una Planta Piloto con Cámara y Arduino
}

\author{
Marcela Cuelloi
}

\section{Resumen}

El presente trabajo desarrolla la implementación de un sistema de medición de la variable caudal utilizando una cámara ip y un microcontrolador Arduino Mega, para ser utilizado en una planta de microfiltrado de líquidos.

Entre diferentes alternativas, este transductor fue implementado a partir de una cámara digital que captura la imagen del flotámetro

La adecuada instrumentación de esta planta permitirá convertirla en un laboratorio remoto para que estudiantes y docentes puedan llevar adelante diversos ensayos a través de una conexión de internet.

Este trabajo fue desarrollado como parte de una Tesis de Grado enmarcada en el proyecto PI 04/I184, de la Facultad de Ingeniería de la Universidad Nacional del Comahue.

Palabras clave: Planta - Laboratorio Remoto - PLC - SCADA - Caudalímetro. 


\section{Abstract}

This work is aimed at measuring the variable flow microfiltration pilot plant by using an IP camera and a microcontroller Arduino Mega. This aims to develop the instrumentation of this plant to turn it into a remote laboratory that allows students and teachers to carry out various tests through an internet connection. This alternative rescues the advantages of virtual laboratories, where it is not necessary to move to it for testing, but with the fundamental difference that the remote laboratory simulation of the process is not done in site, but is monitored remotely results of an actual test.

This work was developed as part of part of an Undergraduate Thesis of Electronic Engineering within the PI 04/I184 project of the Engineering Faculty of tha National University of Comahue.

Keywords: Plant - Remote Laboratory - PLC - SCADA - Flowmeter. 


\section{Introducción}

Uno de los proyectos de investigación que lleva adelante actualmente el Departamento de Electrotecnia de la Facultad de Ingeniería de la Universidad Nacional del Comahue consiste en la implementación de un laboratorio remoto sobre una planta de microfiltración y ultrafiltración del Departamento de Química de la misma facultad. Dicha planta piloto, pensada originalmente para realizar ensayos presenciales, posee membranas encargadas del filtrado y cuenta con diversos elementos de medición que permiten evaluar el comportamiento de los fluidos en el sistema.

El aporte de este proyecto consiste en desarrollar la instrumentación de la planta para convertirla en un laboratorio remoto que permita a estudiantes y docentes llevar adelante diversos ensayos a través de una conexión de internet.

Para la instrumentación de este laboratorio remoto debieron entonces modificarse los elementos de medición y control con los que contaba originalmente la planta piloto, orientada originalmente a ensayos convencionales. De este modo, para poder automatizar el funcionamiento de la planta con un Controlador Lógico Programable (PLC) y a su vez ser supervisada por un sistema de Supervisión, Adquisición y Control de Datos (SCADA), se reemplazaron las válvulas manuales por electroválvulas y se instaló un variador de frecuencia para el control de la bomba impulsora del fluido. Por otra parte también debieron ser acondicionados los medidores de temperatura, presión y caudal con los que cuenta la planta, para que proporcionen una salida eléctrica.

\section{Instrumentación electrónica de los caudalímetros.}

Si bien el reemplazo de los sensores de presión y temperatura fue relativamente directo, en el caso de los caudalímetros no fue tan sencillo. En primer lugar se presentó la consideración del principio de medición del caudalímetro, el elemento primario. Al contar con caudalímetros de área variable instalados, no hubiese sido posible cambiarlos por otro tipo de medidor de caudal, sin modificar las prestaciones y el desempeño de la propia planta piloto (Creus Solé,1997). Así, la opción fue reemplazar los caudalímetros por otros del mismo tipo, pero con salida eléctrica, o bien instalar un transductor adaptado a los sensores ya instalados. Si bien el mismo fabricante de los instrumentos de medición de caudal posee en su catálogo distintos transductores compatibles con estos, se estimó conveniente desarrollar el transductor en la propia Facultad. 


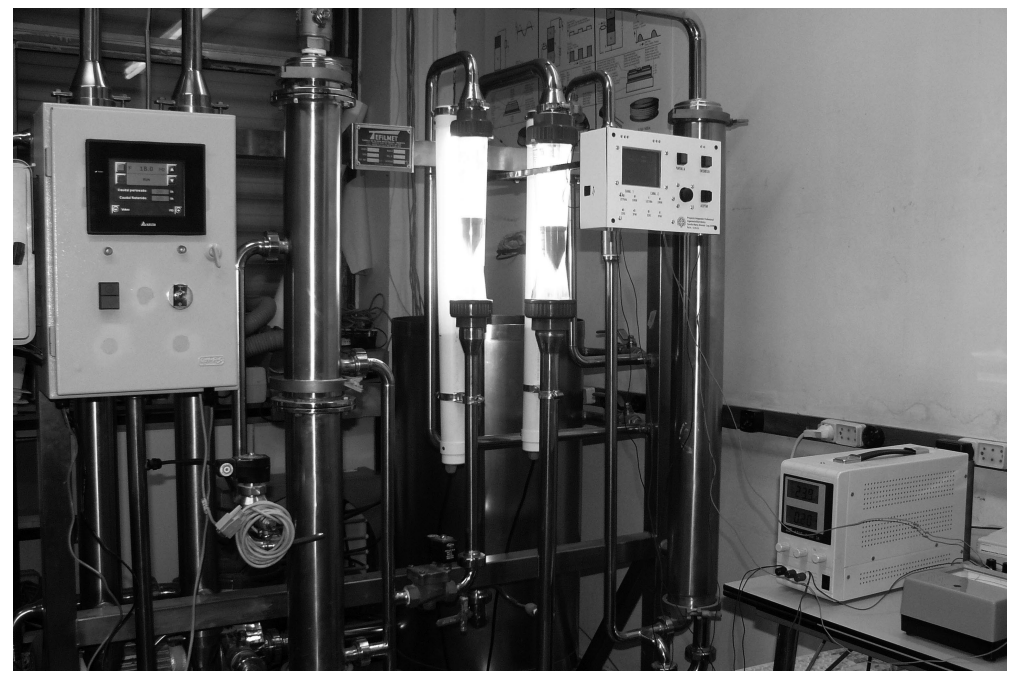

Fig. 1 . Planta piloto con el transductor montado

Se analizaron diferentes alternativas para el mecanismo que utilizaría este transductor. Entre otras, se consideró la posibilidad de modificar el flotante medidor adosándole algún tipo de imán para realizar la medición eléctrica a partir del efecto electromagnético provocado por el movimiento del mismo. Esta primera alternativa fue descartada fundamentalmente porque la modificación de las características del flotante acarrearía una alteración en la calibración del instrumento medidor de caudal.

Otra opción considerada fue la de instalar una serie de barreras ópticas, para cada graduación de la escala, detectando así la posición del flotante, pero también fue desestimada por su poca practicidad, debido a que para obtener una buena precisión de las mediciones se hubieran requerido elementos extras.

Finalmente se definió desarrollar el transductor basándose en una cámara digital, para realizar la captura de la imagen de los caudalímetros y luego por medio de un algoritmo analizar estas imágenes determinando la posición del medidor.

\section{Análisis de imagen}

Existe una gran variedad de algoritmos de reconocimiento de imágenes integrados a distintos paquetes de software, y si bien es importante el desarrollo de los mismos, su implementación más frecuente se limita a las computadoras personales. Es así que, disponiendo de una computadora dedicada específicamente al transductor, existen numerosas alternativas para realizar el reconocimiento en forma relativamente directa. El problema fue que esta posibilidad no representó una solución práctica ya que se buscó que el transductor trabajara como un dispositivo 
autónomo, sin requerir de la asistencia de una computadora. De hecho el transductor fue implementado en base a un microcontrolador que, además de otras funciones, ejecuta el algoritmo para la detección del flotante.

Por otra parte se debió analizar el aspecto de la potencia de cálculo que se requeriría de este procesador. Una implementación directa de los algoritmos clásicos de análisis de imagen involucra un gran volumen de datos, que no podrían manejar microcontroladores de las gamas más económicas. Por esto se optó por desarrollar un algoritmo más simple evitando así el uso de un procesador de costo elevado.

Finalmente se desarrolló un método de detección y análisis de imagen a partir de algoritmos conocidos, ensayando diferentes adaptaciones de ellos para lograr el mejor desempeño.

\section{Elección de la cámara y el microcontrolador.}

El microcontrolador elegido para implementar la programación del transductor fue de la firma Atmel, montado en una placa de desarrollo Arduino MEGA 2560. La ventaja de utilizar una placa de la familia Arduino es la gran diversidad de sensores y periféricos disponibles en el mercado que a su vez cuentan con librerías que permiten utilizar todas sus funciones en forma rápida y directa. Adicionalmente, tener los elementos fundamentales para el funcionamiento del microcontrolador (circuito de reloj, interfaz USB, etc.) ya montados en la placa, permitió reducir de manera importante los potenciales contratiempos durante los ensayos de las diferentes versiones del programa.

Por otra parte, fue necesario contemplar la posibilidad de visualizar la imagen capturada en alguna pantalla para que el usuario pudiera orientar la cámara, facilitar la interacción para otros ajustes disponibles y así capturar correctamente la imagen de los caudalímetros. En un primer momento se analizó utilizar una "Webcam" que se pudiera conectar alternativamente a una computadora, para visualizar la imagen y orientarla, y al microcontrolador para que funcione efectivamente el transductor. Esta opción fue desestimada ya que resultaba ser mucho más conveniente que el dispositivo trabaje en forma totalmente autónoma tanto para las mediciones como para la puesta en funcionamiento.

En función de esto se determinó utilizar un paquete denominado ArduCAM$L F$ que está compuesto por una cámara $O V 2640$, una pantalla LCD color de 3,2 pulgadas y un "shield" que administra la comunicación entre estos dos elementos y el Arduino. 


\section{Algoritmo desarrollado}

El problema fundamental a resolver, fue determinar un algoritmo que permitiera establecer inicialmente la línea de la imagen capturada por la cámara en la que se encuentra el borde superior del flotante que en definitiva es el que indica la medición física del caudalímetro.

En cuanto al sector de la imagen en el que se encuentra el eje vertical del caudalímetro (donde se aplica el algoritmo para determinar la altura del flotante) se optó por dejarlo como un ajuste a realizar por un eventual usuario. Esta solución proporciona además la flexibilidad para que los caudalímetros a medir en los dos canales con los que cuenta el transductor puedan situarse arbitrariamente, siempre que ambos se encuentren dentro del plano de la cámara.

Dado que la cámara capta imagen en colores, inicialmente se consideró la posibilidad de utilizar la información del color para detectar la posición del instrumento pero la medición obtenida de este modo resultó demasiado sensible a las características de la iluminación. Por tal motivo se definió que se trabajaría directamente midiendo el nivel de luminancia, utilizando entonces la información del contraste de la imagen para identificar al flotante. De esta forma se consideraron tres alternativas para maximizar el contraste entre el flotante y el resto del caudalímetro; la primer consideración a tener en cuenta fue la de establecer un fondo homogéneo, y se ensayaron dos alternativas utilizando un fondo blanco y un fondo negro pleno, siempre iluminando desde el frente del caudalímetro. En la figura 2 (a) y (b) se representa la variación de la luminancia de todos los pixeles a lo largo de una columna en el eje vertical del caudalímetro para estas dos alternativas. Si bien en ambas figuras se puede identificar la posición del flotante, el rango de luminancia en el contraste entre los pixeles oscuros y claros no es lo suficientemente grande e inclusive en el caso del fondo negro puede no ser suficiente siquiera para rechazar el ruido de la propia imagen. Por otra parte en estos dos casos, donde la iluminación incidía principalmente desde el frente del caudalímetro, las reflexiones en la superficie del mismo dificultaban aún más la posibilidad de obtener una medición confiable.

Finalmente la solución más apropiada se obtuvo instalando un tubo fluorescente detrás del caudalímetro, coincidente con el eje de medición del transductor. De esta forma se resuelve simultáneamente la necesidad de un fondo homogéneo gracias a la luz difusa del tubo y la necesidad de una iluminación que maximice el contraste entre la zona donde está presente el flotante y el resto de la escala. En la figura 2 (c) se representan los datos obtenidos con esta configuración, donde se puede observar que la diferencia de luminancia entre los puntos claros (con alrededor de 24 puntos) y los oscuros (valores menores a 5) es de mayor magnitud.

Luego, habiendo definido la metodología para realizar la captura con las condiciones más favorables para detectar la posición del flotante, se debió determinar 
el algoritmo de detección propiamente dicho. En la bibliografía existente, uno de los métodos más utilizados para detección de bordes es el Algoritmo de Canny (PAJARES MARTIN SANZ Y CRUZ GARCÍA, 2007). Este algoritmo básicamente plantea una serie de pasos que incluyen en primer lugar el cálculo del gradiente de la luminancia, luego la supresión de los gradientes no máximos ya que esos no corresponderían al contorno de la figura, y finalmente, con esta información, la determinación del contorno en las dos dimensiones conectando los puntos de máximo gradiente. Este algoritmo tal cual está planteado no representaba grandes posibilidades de ser utilizado para el transductor, ya que al trabajar con una imagen en una pequeña franja vertical pierden sentido los cálculos en dos dimensiones. En otras palabras, al no disponer ni necesitar de una representación en dos dimensiones se pierde la posibilidad de relacionar el sentido del gradiente en los distintos puntos para así determinar la forma del contorno.

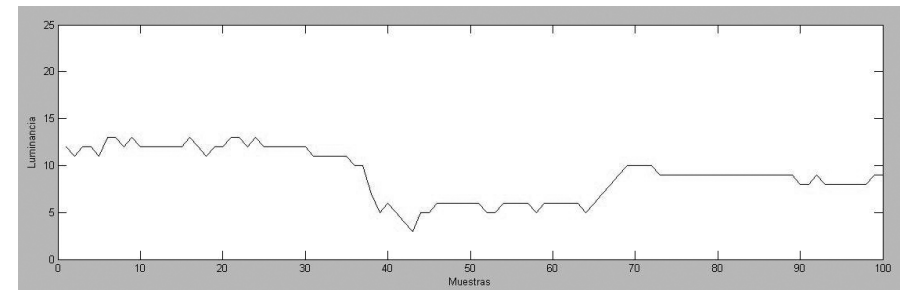

a) Con una superficie blanca de fondo

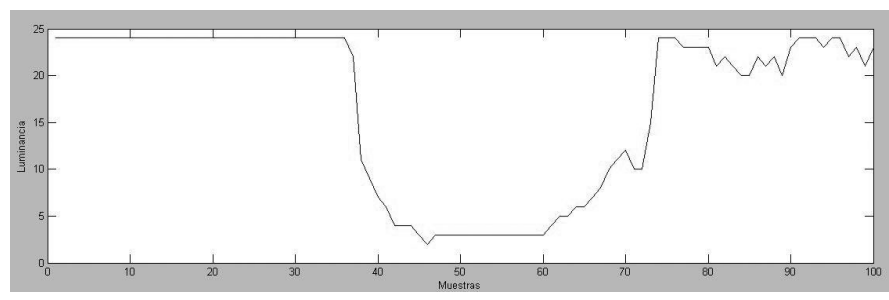

b) Con una superficie negra de fondo

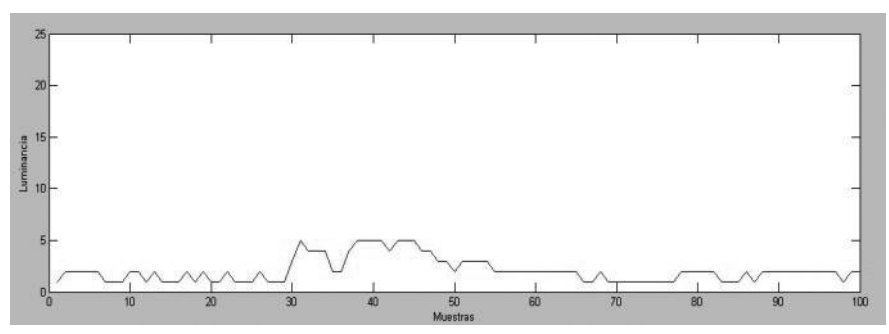

c) Con un tubo fluorescente como fondo

Figura 2: Luminancia para una columna de la captura con diferentes fondos 
Del algoritmo de Canny se tomó la idea de realizar, en lugar del cálculo del gradiente, el cálculo de la diferencia (en una dimensión) para las muestras de una columna y de esa forma obtener una aproximación de la derivada de la luminancia sobre el eje del caudalímetro. De esta forma debería obtenerse el máximo de este valor, en el punto donde se encontrara el borde de la sombra del flotante. Si bien de algún modo se pudo observar un máximo local de la diferencia en torno al borde del flotante, en general nunca es recomendable aplicar un operador de derivada (o de diferencia) a una señal con componentes de ruido (OGATA, 1998). Es así que, en muchos ensayos realizados, no pudo determinarse fehacientemente la posición del flotante a partir de la observación de los resultados obtenidos de esta operación. Los picos de la señal de diferencia debidos al ruido, en algunos casos, eran de una magnitud similar al pico debido a la sombra del flotante.

Por lo tanto se definió finalmente la utilización del propio nivel de luminancia de las muestras de la columna para determinar la posición del flotante. Esto es, definiendo un umbral que discrimine entre pixeles claros y oscuros, y entendiendo que los pixeles oscuros corresponden a la sombra del flotante en la imagen capturada por la cámara. Esta idea, que finalmente es la más sencilla de todas, resultó ser la que proporcionó las mediciones más confiables durante los distintos ensayos que se hicieron en esta etapa. De todas maneras, a esta idea básica se le adicionaron una serie de operaciones para mejorar, de ser posible, la precisión de las medidas.

En primer lugar, los valores de luminancia obtenidos para cada columna se submuestrean, realizando un promedio de 5 pixeles adyacentes; de esta manera se suavizan potenciales pixeles con ruido excesivo que podría afectar a la detección del flotante. Luego, el umbral de decisión no se define como un valor absoluto de luminancia, sino que por el contrario está expresado en términos de una proporción del rango dinámico de luminancia para las muestras de la columna en cuestión. Esto significa que en función del máximo y mínimo de luminancia para la captura de una columna, se determina el valor que permite discernir entre pixeles claros y oscuros. Empíricamente, en los distintos ensayos realizados, se pudo comprobar que los mejores rendimientos se obtuvieron ajustando este umbral simplemente en la mitad del rango dinámico de luminancia. De todos modos, justamente por estar establecido como una proporción del rango, sencillamente puede modificarse una variable en el programa para establecerlo en otro valor, y de hecho el usuario cuenta con esta posibilidad al realizar la configuración inicial del transductor. Cabe aclarar también que esta normalización del umbral, se hace a partir de los máximos y mínimos de luminancia de los pixeles de la columna capturados en el ciclo previo de medición. Esto es porque el microcontrolador utilizado para implementar el algoritmo no tiene suficiente potencia de cálculo para almacenar la totalidad de las muestras de una captura, determinar el máximo y el mínimo, y luego determinar la posición de los pixeles oscuros. Por el contrario debe hacer 
una manipulación de los datos en el mismo momento en que se van descargando de la memoria de la cámara: con cada pixel recibido indaga si pertenece al bloque oscuro en base al umbral determinado con la captura anterior y a su vez retiene los valores máximo y mínimo para actualizar el umbral de la próxima captura. Esto no representa mayores problemas, ya que el periodo entre capturas es de unos pocos segundos y las condiciones de iluminación que podrían afectar al máximo y mínimo de luminancia en general se mantienen constantes.

Además, para otorgar robustez adicional a la medición, la posición de flotante no se determina a partir de encontrar el primer pixel por debajo del umbral, sino que el algoritmo busca un agrupamiento de por lo menos diez pixeles oscuros. De este modo, cualquier pequeño obstáculo que se pueda cruzar delante de la cámara no será confundido con el flotante del caudalímetro. Finalmente, como se cuenta con la captura de tres columnas de la imagen en torno al eje vertical del caudalímetro, se optó por realizar en principio la detección del flotante en cada columna individualmente y luego contrastar entre si los tres valores obtenidos. De este modo, calculando el desvío de estas tres mediciones, se descartan aquellos resultados que no se encuentren dentro de un intervalo aceptable.

\section{Circuito salida analógica}

Para obtener una salida analógica en el rango de 4 a $20 \mathrm{~mA}$, fue necesario diseñar y construir un circuito que proporcionara esta corriente a partir del valor digital medido y proporcionado por el microcontrolador. Para el diseño se tuvieron en cuenta diversas premisas que condicionaron la resolución de distintas características del circuito. En primer lugar se puede destacar la necesidad de que el circuito de salida analógica cuente con elementos de ajuste, para que el usuario pueda ajustar en la forma clásica los controles habituales de "zero" y "span" de cada canal. Esta condición dejó fuera de consideración la posibilidad de utilizar un conversor digital analógico que proporcionara directamente la salida en 4-20mA a partir del valor digital, y en su lugar se utilizó una conversión de dos etapas: en primer lugar un DAC integrado proporciona un valor de tensión a partir del valor digital y en una segunda etapa un arreglo de amplificadores operacionales se utiliza para convertir este valor de tensión en la salida de corriente; es en esta segunda etapa precisamente donde el usuario puede realizar los ajustes mencionados. Un detalle no menor de esta posibilidad para el usuario de realizar los ajustes, es que el ajuste de los dos parámetros debe ser independiente en al menos uno de los sentidos; es decir, que el ajuste del span no altere el equilibrio del zero. La otra condición fundamental fue la de que el transductor no requiera fuentes adicionales, es decir que todos los elementos del transductor puedan alimentarse a partir de la misma fuente de $24 \mathrm{~V}$ 
que alimentaría al eventual PLC que supervise el funcionamiento del transductor. Esto permite una mayor practicidad en la utilización del transductor y evita sumar una eventual fuente de ruido. Como contrapartida dejó fuera de lugar la forma clásica de alimentar el circuito de amplificadores operacionales por medio de fuentes simétricas, en cambio, se debió trabajar con distintas tensiones de referencia en cada AO para lograr el funcionamiento correcto.

Cumpliendo estas premisas, se logró diseñar un circuito que funcionó satisfactoriamente, resultando una salida del tipo NPN a 3 hilos. Cabe destacar que el circuito diseñado además permite trabajar con un amplio rango de resistencias de trabajo en el lazo de salida y más allá de que todo el transductor puede ser alimentado con una misma fuente de alimentación, los lazos de ambos canales son independientes.

\section{Conclusiones}

La conclusión fundamental del trabajo desarrollado es que efectivamente fue posible implementar un transductor para el caudalímetro de área variable en base a la captura de la imagen por una cámara digital. Esta alternativa para detectar la medición de un caudalímetro de este tipo presenta algunas limitaciones. Es necesario establecer un espacio libre entre la cámara y los caudalímetros, de modo que estos puedan ser capturados dentro del campo visual. Por otra parte, la fiabilidad de la medición puede ser afectada por las condiciones de iluminación en el ambiente, tal es así que para la utilización del transductor desarrollado en este trabajo, fue necesario establecer un elemento de iluminación auxiliar.

Sin embargo este método posee, como contrapartida, la ventaja de que el transductor puede utilizarse sin realizar ningún tipo de modificación al caudalímetro en sí. A su vez, el transductor desarrollado, puede utilizarse sin mayores modificaciones en caudalímetros de área variable de distintas marcas o modelos; siempre que sea posible aproximar la escala de medición por la misma ley cuadrática que se utiliza en este trabajo la aplicación será directa, independientemente de los valores absolutos de la escala y su tamaño. En caso contrario, tampoco representaría un impedimento, ya que con una sencilla modificación del programa cargado en el microcontrolador podría adaptarse.

En lo que respecta al algoritmo elaborado para detectar la posición del flotante, y a partir de las pruebas realizadas, se evalúa como satisfactorio el desempeño del mismo. La decisión de desarrollar un algoritmo simple, para así poder implementarlo en un procesador de moderadas prestaciones, no representó una limitación en el funcionamiento final del transductor.

Durante el proceso de elaboración del algoritmo se ensayaron, en base a algunos 
de los métodos de análisis de imagen más conocidos, diferentes operaciones sobre las muestras de la captura que permitieran la detección de la posición del flotante. Estas distintas operaciones de filtrado y derivación, en base al cálculo de ecuaciones en diferencias, además de significar un costo de cómputo mayor para el procesador, en la comparación global de los resultados para los ensayos realizados no obtuvieron desempeños alentadores. Finalmente, en el proceso de buscar una mejoría en el rendimiento, esta se obtuvo paradójicamente simplificando el principio de análisis. Tal es así que el algoritmo desarrollado utiliza un principio elemental para determinar la medición del caudalímetro; evalúa el nivel de iluminación de los pixeles de la imagen capturada. En esta misma línea de pensamiento, al definir el umbral de decisión para este parámetro, se obtuvieron los mejores resultados precisamente asignándole un valor del 50\% del rango dinámico de luminancia.

En referencia al hardware, y particularmente a la utilización de una placa Arduino, se destaca la ventaja de este tipo de herramientas para el proceso de desarrollo de un prototipo. Por trivial que parezca, el hecho de que en dicha placa estén montados de fábrica los elementos básicos para el funcionamiento del microcontrolador (circuito de reloj, alimentación, interfaz USB) agiliza notablemente el proceso de ensayo y depuración de las distintas versiones del programa. Por otra parte, la gran inserción de esta familia de placas en el mercado, ha traído aparejada la proliferación de una variada gama de sensores y accesorios para las mismas. Este desarrollo, a su vez, ha posibilitado la disponibilidad de infinidad de librerías ya desarrolladas bajo una licencia abierta para la utilización de sus diversas funciones y periféricos.

Como contrapartida a las ventajas detalladas en el párrafo precedente, es preciso señalar que esta misma comodidad que proveen las funciones ya empaquetadas, sólo es tal cuando se intenta resolver un problema tipo. Por el contrario, cuando se necesita realizar alguna operación fuera de lo común esta ventaja desaparece, e inclusive en algunos casos, debido al mismo "empaquetado", la resolución puede ser aún más complicada que si se realizara desde la fuente.

Por otra parte, la practicidad de estas placas prefabricadas tiene un costo aparejado, el precio de una placa Arduino supera notablemente al del microcontrolador individual que la integra. Es por ello que si bien la utilización de estas placas es recomendable para el desarrollo de un prototipo, una vez desarrollado el modelo final es conveniente elaborar una placa dedicada específicamente donde se instalen el procesador y los periféricos necesarios. 


\section{Bibliografía}

CREUS SOLÉ, ANTONIO, Instrumentación industrial, Sexta edición, Marcombo S. A., Barcelona, España, 1997

PAJARES MARTINSANZ, GONZALO y CRUZ GARCÍA, JESÚS M. DE LA, Visión por computador. Imágenes digitales y aplicaciones, Segunda edición, Ra-Ma S. A. Editorial y Publicaciones, España, 2007.

OGATA, KATSUHIKO, Ingeniería de control moderna, Tercera edición, PrenticeHall Hispanoamericana S. A., México, 1998

http://www.arducam.com

http://www.arduino.cc 